\title{
Quit smoking improves gastroesophageal reflux symptoms and quality of life
}

\author{
Kou Nakajima ${ }^{1}$, Akihito Nagahara $^{1 *}$, Akihiko Kurosawa $^{1}$, Kuniaki Seyama $^{2}$, Daisuke Asaoka ${ }^{1}$, \\ Taro Osada ${ }^{1}$, Mariko Hojo ${ }^{1}$, Sumio Watanabe ${ }^{1}$ \\ ${ }^{1}$ Department of Gastroenterology, Juntendo University School of Medicine, Tokyo, Japan; \\ *Corresponding Author: nagahara@juntendo.ac.jp \\ ${ }^{2}$ Department of Respiratory Medicine, Juntendo University School of Medicine, Tokyo, Japan.
}

Received 11 September 2011; revised 6 October 2011; accepted 21 October 2011.

\section{ABSTRACT}

Background: Smoking is considered to be risk factors of gastroesophageal reflux disease (GERD). The present study aimed to reveal whether quit smoking improves GERD symptoms and QOL of patients. Methods: In this prospective study, 33 patients who participated in a 12-week quit smoking program filled out the Frequency Scale for the Symptoms of GERD (FSSG) questionnaire, and SF8 QOL questionnaire. Patients filled out the questionnaires at baseline and during the program at 2 weeks, 4 weeks, 8 weeks and 12 weeks. In the FSSG, the responses were scored and the reflux score (RS), dysmotility score (DS) and total score (TS) were calculated. Results: There were 22 males and 11 females. Their mean age was $54.8 \pm 13.0$ (mean \pm SD) yr, BMI was $22.9 \pm 4.0$, and duration of smoking was $33.5 \pm 12.5$ years. Ten patients belonged to GERD subgroup (baselineFSSGscore $\geq 8$ ). All patients were successful at quit smoking. Scores of TS/RS/DS are $8.6 \pm 1.8$ (mean \pm $\mathrm{SE} / 4.2 \pm 0.9 / 4.5 \pm 0.9$ at baseline, $4.7 \pm 1.6^{* *} / 2.5 \pm$ $0.9^{* *} / 2.3 \pm 0.7^{* *}$ at $2 \mathrm{w}, 5.7 \pm 1.3^{\star *} / 2.6 \pm 0.6^{*} / 3.0 \pm$ $0.7^{*}$ at $4 \mathrm{w}, 4.5 \pm 1.4^{*} / 2.2 \pm 0.8^{*} / 2.3 \pm 0.8^{*}$ at $8 \mathrm{w}$ and $3.7 \pm 1.2^{* *} / 1.7 \pm 0.6^{* *} / 2.0 \pm 0.7^{* *}$ at $12 \mathrm{w}$, respectively $\left({ }^{* *} p<0.01,{ }^{*} p<0.05\right.$ vs. baseline). Among GERD subgroup, Scores of TS/RS/DS are 18.0 $\pm 2.9 / 8.9 \pm 1.6 / 9.1 \pm 1.5$ at baseline, $8.8 \pm$ $3.0 / 5.1 \pm 1.7 / 3.7 \pm 1.6$ at $2 \mathrm{w}, 10.8 \pm 2.9 / 5.4 \pm$ $1.6 / 5.4 \pm 1.5$ at $4 \mathrm{w}, 7.6 \pm 2.9^{*} / 4.1 \pm 1.6 / 3.5 \pm 1.5^{*}$ at $8 \mathrm{w}$ and $7.1 \pm 2.9^{\star} / 3.2 \pm 1.6^{*} / 3.9 \pm 1.5^{*}$ at $12 \mathrm{w}$, respectively. Regarding QOL, physical component score has significantly improved at 2, 4, 8 and $12 \mathrm{w}$ and mental component score at $4 \mathrm{w}$, respectively. Conclusions: Quit smoking significantly improved not only GERD symptoms but also QOL, indicating that quit smoking might be an option in the treatment strategy of GERD symptoms.

Keywords: Quit Smoking; FSSG; GERD; SF8

\section{INTRODUCTION}

Gastrointestinal (GI) symptoms are known to be related to the habit of smoking, which is reportedly an aggravating factor of especially GERD symptoms including heartburn [1-3]. Quit smoking is well known to have positive effect on personal health, on the contrary, some reports described that quit smoking brought about insomnia, obesity, restlessness, stress, or psychological instability [4] and was followed by constipation [5]. However, there has been no report describing the relationship between quit smoking and GERD symptoms. To reveal whether quit smoking could improve GERD symptoms and QOL, patients who participated in the quit smoking program at the outpatient clinic of the Department of Respiratory Medicine in Juntendo University Hospital were asked to fill out the Frequency Scale for the Symptoms of GERD (FSSG) questionnaire and SF8 QOL questionnaire.

\section{METHODS}

In this prospective study, patients who joined a 12week quit smoking program at the outpatient clinic of the Department of Respiratory Medicine in Juntendo University Hospital between June 2008 and April 2009, were asked to fill out the FSSG questionnaire as well as the SF8 QOL questionnaire. All patients were not administered proton pump inhibitors.

The FSSG was designed to evaluate GERD symptoms in the Japanese population. The FSSG consists of 12 items; 7 items concern gastric acid reflux and 5 items 
concern dysmotility symptoms (Figure 1). Patients were asked to rate each item on a scale of 0 to 4 . Comparisons of the scores were made for each item between before and after starting (at 2, 4, 8, and 12 weeks) the quit smoking program. The RS was calculated as the sum of the scores of the 7 items concerning gastric acid reflux, and DS was calculated as the sum of the scores of the 5 items concerning dysmotility symptoms. TS was calculated as the sum of the scores of all 12 items. The SF8 QOL questionnaire was composed of the physical component score (PCS) and mental component score (MCS) and included 8 items. Similar to the FSSG, the scores were compared for each item between before and after starting (at 2, 4, 8, and 12 weeks) the quit smoking program.

Patients in the quit smoking program were initially prescribed varenicline tartrate. In patients who could not tolerate varenicline tartrate, it was changed to Nicotine Patch. Varenicline tartrate was taken at a daily dose of 1 tablet containing $0.5 \mathrm{mg}$ from day 1 to 3, 1 tablet twice a day from day 4 to 7 , and 2 tablets containing $1 \mathrm{mg}$ in each twice a day thereafter.
Data are expressed as the mean \pm standard deviation in patients' characteristics and QOL scores, and mean \pm standard error in FSSG scores. Data were analyzed with the Wilcoxon test. Physicians explained in detail the purpose of this study to each patient and obtained informed consent from them.

\section{RESULTS}

Thirty-three patients in the 12-week quit smoking program were enrolled in the present study. There were 22 males and 11 females. Their age was $54.8 \pm 13.0 \mathrm{yr}$, BMI was $22.9 \pm 4.0$. Duration of smoking was $33.5 \pm$ 12.5 years and number of daily smoked cigarettes was $26.0 \pm$ 11.9/day. The Brinkman index was 902.6 \pm 561.4. The Brinkman index was calculated by multiplying the number of years of smoking and the number of daily smoked cigarettes. Seventeen patients received varenicline tartrate, while the other 16 patients were switched to a nicotine patch. Regarding underlying disease, 2 patients suffered from chronic obstructive pulmonary disease, 3 patients suffered from asthma and 2 patients suffered from hypertension.

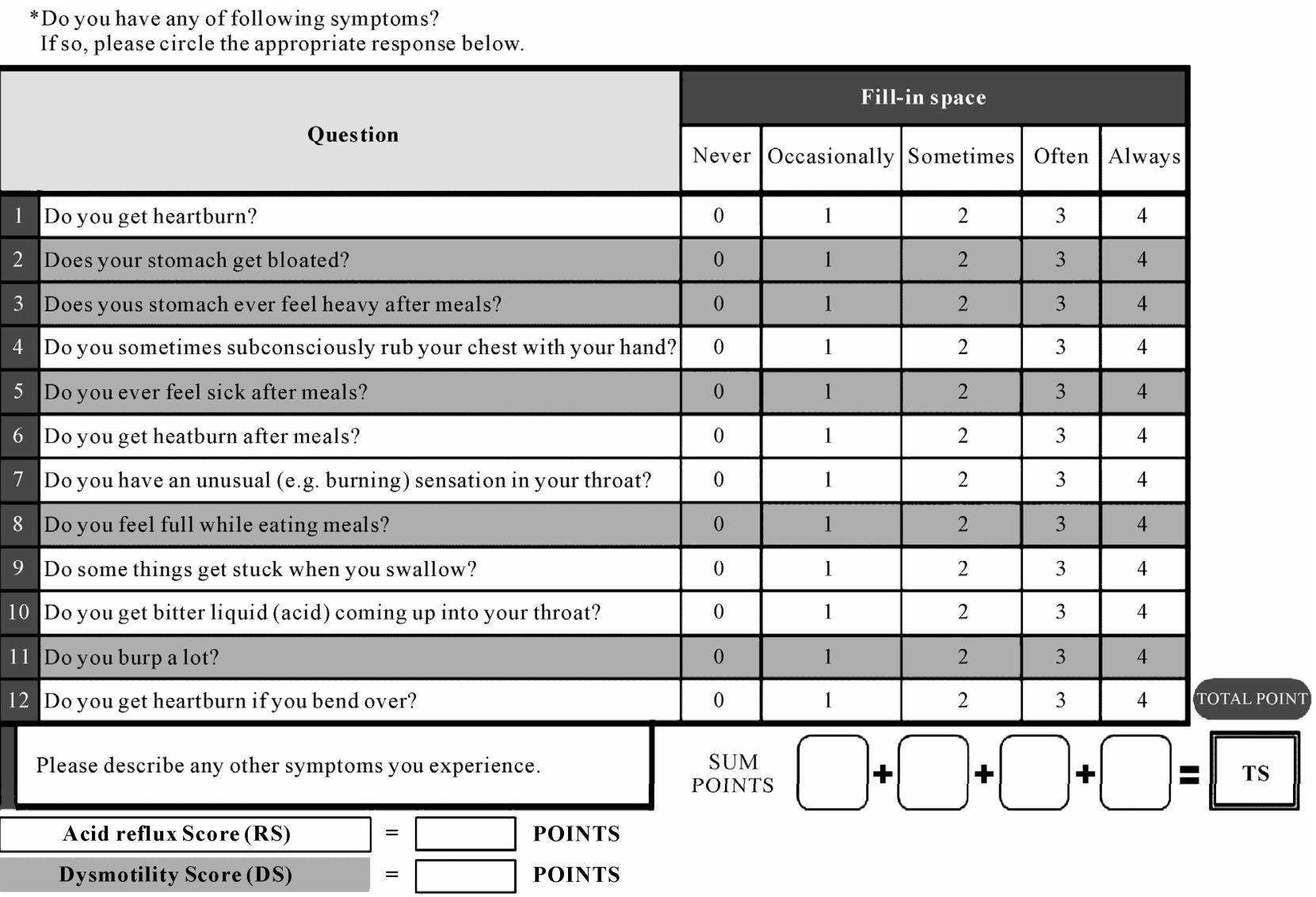

Figure 1. Frequency Scale for the Symptom of GERD (FSSG) questionnaire is diagnostic tool of GERD consisting of 12 items that are most commonly being complained by GERD patients and it has 7 questions related to reflux symptoms and 5 questions related to dysmotility symptoms. 
Four of the 33 patients were excluded from the analysis due to missing data in the baseline FSSG questionnaire. Of the remaining all 29 patients succeeded in quit smoking. Patients who did not fill out the FSSG questionnaires completely were excluded from the analysis of TS, DS, and RS. Data analyses on TS, DS, and RS were performed on 22 patients at 2 weeks, 25 patients at 4weeks, 23 patients at 8 weeks, and 23 patients at 12 weeks. Evaluation of the responses to each questions of the FSSG was performed on individual patients who answered each question at 2, 4, 8, and 12 weeks.

In the FSSG before quit smoking, bloat, feeling heavy in the stomach after meals, unpleasant sensation in the throat, and burps were commonly complained as $50 \%$ or more of the patients as shown in Figure 2.

Regardless severity of smoking habit (duration of smoking, the number of daily smoked cigarettes and Brinkman index), GERD symptoms after quit smoking were improved.

TS at baseline was $8.6 \pm 1.8$, and significant improvement was seen at 2 weeks $(4.7 \pm 1.6$, p $<0.01)$, 4 weeks $(5.7 \pm 1.3$, p $<0.01)$, 8 weeks $(4.5 \pm 1.4$, p $<0.05)$ and 12 weeks $(3.7 \pm 1.2$, $\mathrm{p}<0.01)$ compared with that at baseline (Figure 3(a)). RS at baseline was $4.2 \pm 0.9$, and significant improvement was seen at 2 weeks $(2.5 \pm 0.9$, $\mathrm{p}<0.01)$, 4 weeks $(2.6 \pm 0.6, \mathrm{p}<0.05), 8$ weeks $(2.2 \pm$ $0.8, \mathrm{p}<0.05)$ and 12 weeks $(1.7 \pm 0.6, \mathrm{p}<0.01)$ compared with that at baseline (Figure $\mathbf{3 ( b ) )}$. DS at baseline was $4.5 \pm 0.9$, and significant improvement was seen at
2 weeks $(2.3 \pm 0.7, \mathrm{p}<0.01)$, 4weeks $(3.0 \pm 0.7, \mathrm{p}<$ $0.05), 8$ weeks $(2.3 \pm 0.8, \mathrm{p}<0.05)$ and 12 weeks $(2.0 \pm$ $0.7, \mathrm{p}<0.01$ ) compared with that at baseline (Figure 3(c)). In this program, 15 received varenicline tartrate and the other 14 patients received a nicotine patch because of intolerance to varenicline tartrate. However, as for FSSG score, there was no significant difference between varenicline tartrate and nicotine patch group at the end of this program.

Regarding the change of scores of each item in FSSG, all items in the FSSG except for Q8 ("feel full while eating meals”) and Q9 (“stuck”) significantly improved during the program. Particularly, significant improvements were observed in Q3 (“feel heavy after meals"), Q7 (“unusual sensation in the throat”), Q10 ("acid into the throat”), and Q11 ("burp”) at only 2 weeks after starting the program and thereafter (Figure 4).

Since cutoff score of FSSG is 8 points [6], we analyzed GERD subgroup $(n=10)$ whose score $\geq 8$. TS at baseline was $18.0 \pm 2.9$, and significant improvement was seen at 8 weeks (7.6 \pm 2.9 , p < 0.05), 12 weeks $(7.1$ \pm 2.9 , p $<0.05$ ) compared with that at baseline (Figure 5(a)). RS at baseline was $8.9 \pm 1.6$, and significant improvement was seen at 12 weeks $(3.2 \pm 1.6$, p $<0.05)$ compared with that at baseline (Figure 5(b)). DS at baseline was $9.1 \pm 1.5$, and significant improvement was seen at 8 weeks $(3.5 \pm 1.5, \mathrm{p}<0.05)$ and 12 weeks $(3.9 \pm$ $1.5, \mathrm{p}<0.05)$ compared with that at baseline (Figure 5(c)).

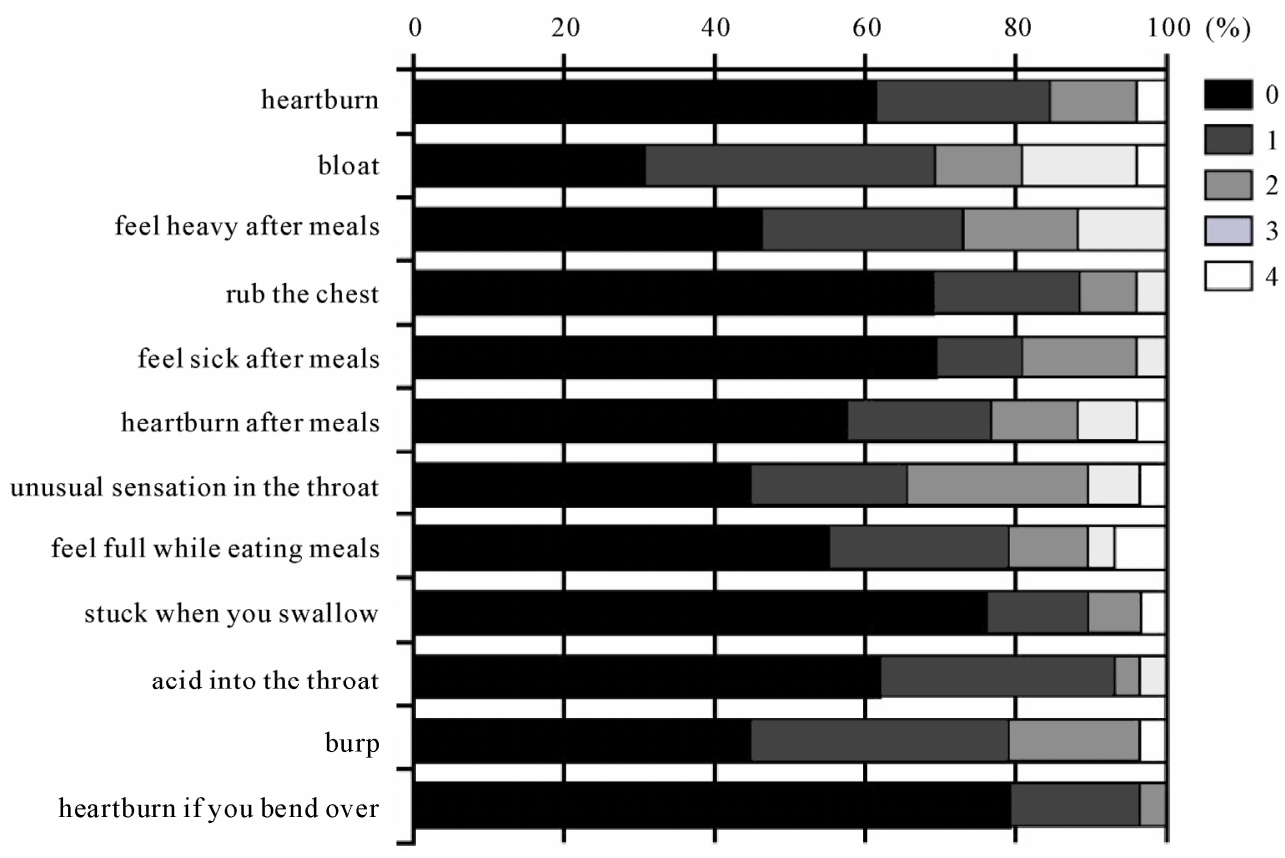

Figure 2. Distributions of FSSG score at baseline. In the FSSG before quit smoking, bloat, feeling heavy in the stomach after meals, unusual sensation in the throat, and burps were commonly complained as $50 \%$ or more of the patients. 

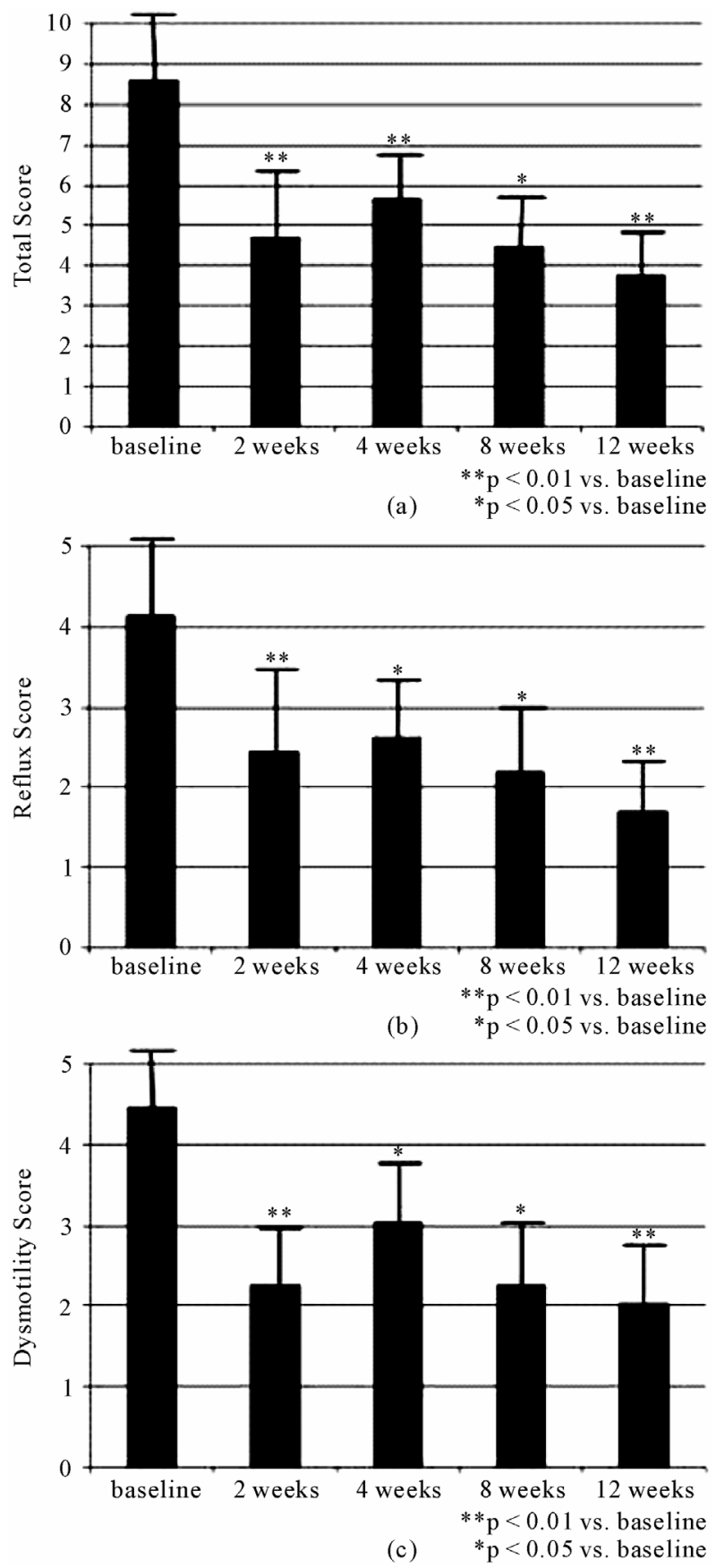

Figure 3. Change of FSSG-Total score (TS) by quit smoking program (a) upper panel. TS showed significant improvement at 2 weeks ( $p<0.01)$, 4 weeks ( $p<0.01) 8$ weeks $(p<0.05)$ and 12 weeks $(\mathrm{p}<0.01)$ compared with that at baseline, respectively. Change of FSSG-Reflux score (RS) by quit smoking program (b) middle panel. RS showed significant improvement at 2 weeks ( $\mathrm{p}<0.01)$, 4weeks $(\mathrm{p}<0.05) 8$ weeks ( $\mathrm{p}$ $<0.05)$ and 12 weeks $(\mathrm{p}<0.01)$ compared with that at baseline, respectively. Change of FSSG-Dysmotility score (DS) by quit smoking program (c) lower panel. DS showed significant improvement at 2 weeks $(\mathrm{p}<0.01), 4$ weeks $(\mathrm{p}<0.05) 8$ weeks $(\mathrm{p}<0.05)$ and 12 weeks $(\mathrm{p}<0.01)$ compared with that at baseline, respectively.
As shown in Figure 6, PCS/MCS in SF8 QOL score was $47.3 \pm 5.4 / 48.9 \pm 6.6$ at baseline, $50.6 \pm 4.9 * / 51.1 \pm$ 6.7 at 2 weeks, $51.2 \pm 4.7^{* * / 51.1} \pm 4.6^{*}$ at 4 weeks, 51.3 $\pm 5.8 * * / 50.7 \pm 5.2$ at 8 weeks and $50.6 \pm 5.6 * / 50.2 \pm 7.0$ at 12 weeks, respectively $\left({ }^{* *} \mathrm{p}<0.01,{ }^{*} \mathrm{p}<0.05\right.$ vs. baseline). PCS showed significant improvements at 2 to 12 weeks after quitting smoking compared with those at baseline, while MCS improved at 4 weeks. There was no significant difference in PCS/MCS score between varenicline tartrate and nicotine patch group at the end of this program.

\section{DISCUSSION}

Nicotine, when rapidly absorbed into the body of nicotine-naïve people, acts on parasympathetic ganglions as well as cholinergic nerve endings, resulting in stimulation of peristaltic movement of the GI tract and thus bringing on nausea and diarrhea [7]. In contrast, chronic long-term ingestion of nicotine alters the peristaltic movement of the GI tract, influences the function of multiple endocrine organs, and injures defense mechanisms of the GI tract [8]. It also induces the release of mediators as well as hormones that affect the structural integrity of the epithelium and mucosa $[9,10]$. Furthermore, reducing LES pressure may result in symptom generation of GERD [11]. Kahrilas PJ et al. described that the mechanisms of acid reflux during cigarette smoking were mainly dependent on the coexistence of diminished LES pressure and the majority of acid reflux occurred with coughing or deep inspiration during which abrupt increases in intra-abdominal pressure overpowered a feeble sphincter rather than transient LES relaxation [12]. Accordingly, cigarette smoking may correlate GERD symptom through various mechanisms.

Kusano et al. [6] reported the FSSG to evaluate GERD symptoms. They reported that it showed a sensitivity of $62 \%$, specificity of $59 \%$, and accuracy of $60 \%$ when the cutoff score was set at 8 points and FSSG is widely used to evaluate GERD symptoms thereafter [13-15]. In this study, mean score of FSSG before starting the program was 8.6 and $30 \%$ of patients was equal or more than 8 points. According to the recent review form Japan, ratio of GERD was reported as 1.3 to $13.7 \%$ in general population [16]. Although the patient number of this study is a few, ratio of GERD might be more frequent than expected among smokers. Intervention studies with patients with GERD symptoms, RS and DS are ameliorating associated with PPI administration $[17,18]$. Consequently, the FSSG is useful for evaluation of GERD symptoms and therapeutic effect.

GERD consists of three concept, which are reflux esophagitis (RE) with symptoms, reflux symptoms without RE and RE without symptoms [16]. Upper GI endo- 


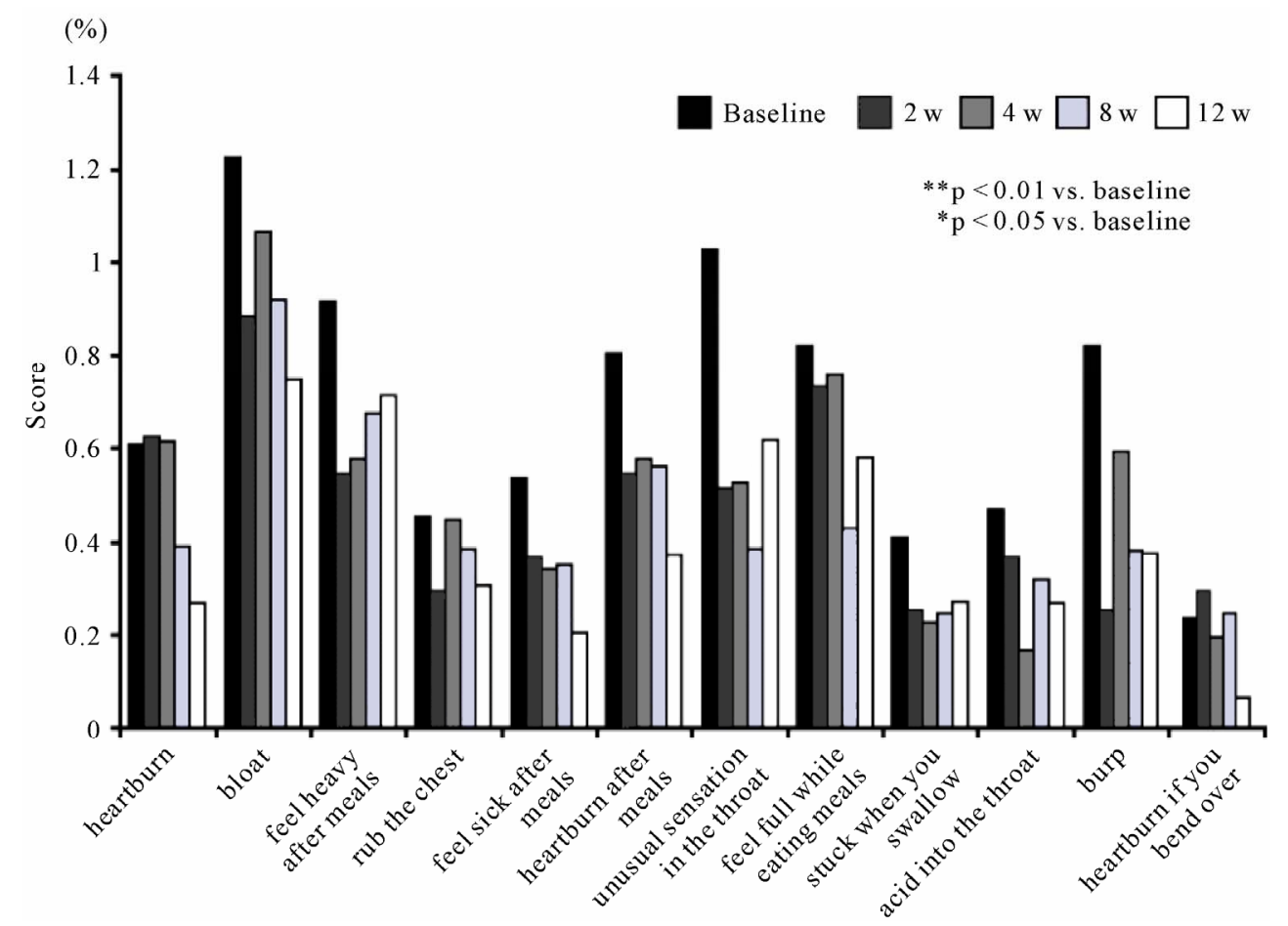

Figure 4. Change of scores of each item in FSSG, all items in the FSSG except for "feel full while eating meals" and "stuck when you swallow" significantly improved during the program. Particularly, significant improvements were observed in "feel heavy after meals", "unusual sensation in the throat", "acid into the throat" and "burp” at only 2 weeks after starting the program and thereafter.

scopy is necessary to diagnose RE, however, general physicians who don't have endoscopic equipment should deal with GERD patients. Therefore, some study was focused on uninvestigated GERD, which is diagnosed by symptoms [17,19]. Indeed, GERD management guideline which was published by The Japanese Society of Gastroenterology described that initial therapy could be performed without endoscopy for patient with GERD symptoms [20]. Accordingly, control of GERD symptoms should be clinical significance regardless endoscopic findings. TS, RS, and DS were improved in all patient in this study (Figure 3). Surprisingly, GERD subgroup whose FSSG score $\geq 8$ reveled that FSSG score significantly improved at 12 week (Figure 5). Among these patients group, $12.5 \%, 11.0 \%, 33.3 \%$ and $40.0 \%$ of these recognized complete symptom relief at 2, 4, 8 and 12 week respectively. These result suggested that quit smoking may be an option of treatment of GERD symptoms, or at least lead of quit smoking for patients with GERD symptom could have additive therapeutic effect.

Quit smoking, as evaluated with the FSSG, improved the following GERD symptoms promptly only 2 weeks; "feeling heavy after meals", "acid in the throat" and "burp". This was because quit smoking might improve the nicotine-induced decline in esophageal lower sphincter pressure, which had led to frequent occurrence of reflux symptoms [3,11,12]. Quit smoking also alleviated the "unusual sensation in your throat" in a short period of time in this study. Chang-Chun Lin et al. [21] and Belafsky P.C. et al. [22] pointed out in their studies using RSI (reflux symptom indexes) scores that smoking provoked symptoms related to the vocal cord or the laryngopharynx. Therefore, quit smoking seemed to ease such symptoms.

Our result indicated that improvement of GERD symptoms brought about by quit smoking was not related to Brinkman index. Hence, it seems that even a heavy smoker would benefit from quit smoking in terms of improvement of GERD symptoms.

There are reports that indicated that quit smoking improved self-control and health transition and that quit smoking improved the QOL of asthmatic ex-smokers $[23,24]$. In this study showed the similar result as these previous studies. Especially, PCS kept improving significantly during the program. Moreover, both PCS and MCS exceeded 50 points which is mean Japanese national level after starting the program at only 2 weeks and maintained until the end of program. In this study, quit smoking led prompt improvement in some symptoms, might direct impact on QOL particularly PCS which is concerning physical health.

In this study, it is unclear whether patients had reflux 


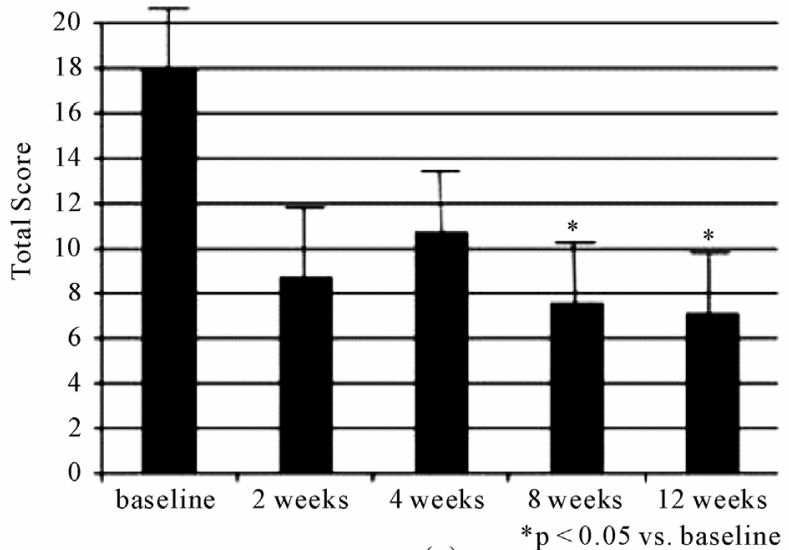

(a)

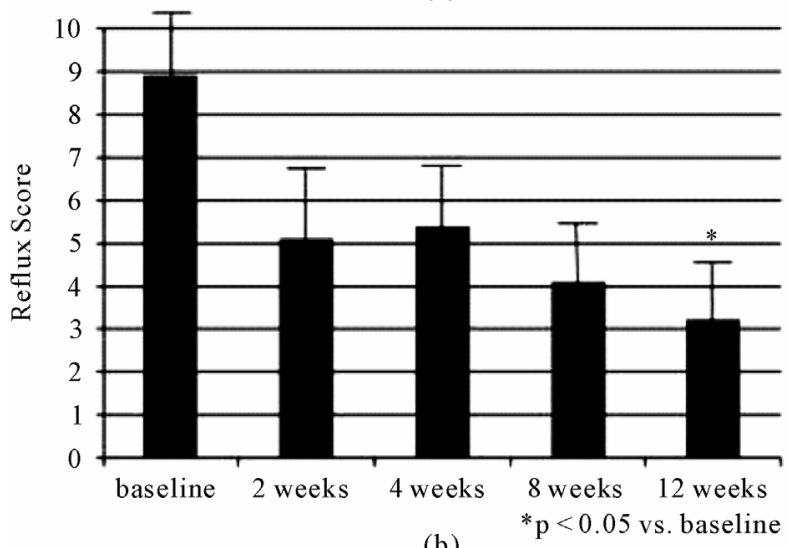

(b)

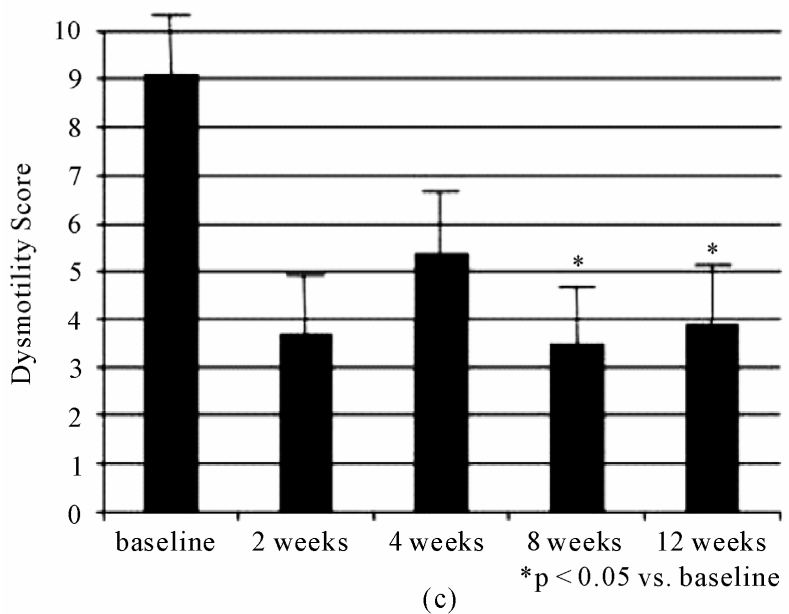

Figure 5. Change of FSSG-Total score (TS) by quit smoking program among GERD subgroup (a) upper panel. TS showed significant improvement at 8weeks $(\mathrm{p}<0.05)$ and 12 weeks ( $<0.05$ ) compared with that at baseline, respectively. Change of FSSG-Reflux score (RS) by quit smoking program among GERD subgroup (b) middle panel. RS showed significant improvement at 12 weeks $(\mathrm{p}<0.05)$ compared with that at baseline, respectively. Change of FSSG-Dysmotility score (DS) by quit smoking program among GERD subgroup (c) lower panel. DS showed significant improvement at 8 weeks $(\mathrm{p}<0.05)$ and 12 weeks ( $p<0.05)$ compared with that at baseline, respectively.

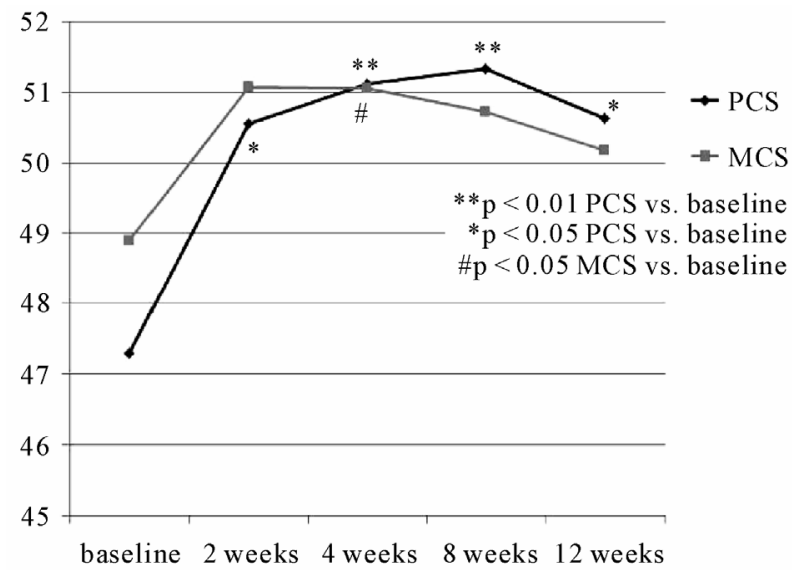

Figure 6. Change of SF8 QOL by quit smoking program. Physical component summary (PCS) significant improved at 2, 4,8 , and 12 weeks versus baseline. Mental component summary (MCS) significant improved at 4 weeks versus baseline. Both PCS and MCS exceeded 50 points promptly after starting the program at only 2 weeks and maintained until the end of program.

esophagitis or peptic ulcer since we did not perform an endoscopy. We focused on change of "symptoms" of GERD by quit smoking program and revealed its improvement. Moreover, it remains to be studied whether quit smoking induced improvement of symptoms is maintained for a long period of time since our follow-up period was short. These points are limitation of this study. We hope that a large-scale long-term study will be conducted in the future.

In Conclusions, Quit smoking improved not only GERD symptoms but also QOL. We showed that quit smoking has the potential of being one of the therapeutic strategic options for GERD symptom treatment.

\section{CONFLICT OF INTEREST}

There is no conflict of interest.

\section{REFERENCES}

[1] Salter, R.H. (1974) Occasional Survey: Lower oesophageal sphincter. Therapeutic implications. Lancet, 7853, 347-349. doi:10.1016/S0140-6736(74)93088-8

[2] Stanciu, C. and Bennett, J.R. (1972) Smoking and Gstroo-oesophageal reflux. British Medical Jounal, 30, 793-795.doi:10.1136/bmj.3.5830.793

[3] Chattopadhyay, D.K., Greaney, M.G. and Irvin, T.T. (1977) Effect of cigarette smoking on the lower oesophageal sphincter. Gut, 18, 833-835. doi:10.1136/gut.18.10.833

[4] Gritz, E.R., Carr, C.R. and Marcus, A.C. (1991) The tobacco withdrawal syndrome in unaided quitters. British Journal of Addiction, 86, 57-69.

[5] Hajek, P., Gillison, F. and McRobbie, H. (2003) Stopping smoking can cause constipation. Addiction, 98, 1563- 
1567. doi:10.1046/j.1360-0443.2003.00497.x

[6] Kusano, M., Shimoyama, Y., Sugimoto, S., et al. (2004) Development and evaluation of FSSG: frequency scale for the symptoms of GERD. Journal of Gastroenterology, 39, 888-891. doi:10.1007/s00535-004-1417-7

[7] Massarrat, S. (2008) Smoking and Gut. Archives of Iranian Medicine, 11, 293-305

[8] Murthy, S.N., Dinosi, V.P. Jr, Clearfield, H.R. and Chey, W.J. (1977) Simultaneous measurement of basal pancreatic, gastric acid secretion, plasma gastrin, and secretin during smoking. Gastroenterology, 73, 758-761.

[9] Miller, T.A. (1983) Protective effects of prostaglandins against gastric mucosal damage: current knowledge and proposed mechanisms. American Journal of Physiology, 245, G601-G623.

[10] Quimby, G.F., Bonnice, C.A., Burstein, S.H. and Eastwood, G.L. (1986) Active smoking depresses prostaglandin synthesis in human gastric mucosa. Annals of Internal Medicine, 104, 616-619.

[11] Smi,t C.F., Copper, M.P., van Leeuwen, J.A., Schoots, I.G. and Stanojcic, L.D. (2001) Effect of cigarette smoking on gastropharyngeal and gastroesophageal reflux. Annals of Otology, Rhinology, and Laryngology, 110, 190-193.

[12] Kahrilas, P.J., Gupta, R.R. (1990) Mechanisms of acid reflux associated with cigarette smoking. Gut, 31, 4-10. doi:10.1136/gut.31.1.4

[13] Miyamoto, M., Haruma, K., Takeuchi, K. and Kuwabara, M. (2008) Frequency scale for symptom of gastroesophageal reflux disease predicts the need for addition of prokinetics to proton pump inhibitor therapy. Journal of Gastroenterology and Hepatology, 23, 746-751. doi:10.1111/j.1440-1746.2007.05218.x

[14] Oridate, N., Takeda, H., Mesuda, Y., et al. (2008) Evaluation of upper abdominal symptoms using the Frequency Scale for the Symptoms of Gastroesophageal Reflux Disease in patients with laryngopharyngeal reflux symptoms. Journal of Gastroenterology, 43, 519-523. doi:10.1007/s00535-008-2189-2

[15] Danjo, A., Yamaguchi, K., Fujimoto, K., et al. (2009) Comparison of endoscopic findings with symptom assessment systems (FSSG and QUEST) for gastroesophageal reflux disease in Japanese centres. Journal of Gastroenterology and Hepatology, 24, 633-638. doi:10.1111/j.1440-1746.2008.05747.x
[16] Fujiwara, Y. and Arakawa, T. (2009) Epidemiology and clinical characteristics of GERD in the Japanese population. Journal of Gastroenterology, 44, 518-534. doi:10.1007/s00535-009-0047-5

[17] Nagahara, A., Asaoka, D., Hojo, M., et al. (2010) Observational comparative trial of the efficacy of proton pump inhibitors versus histamine-2 receptor antagonists for uninvestigated dyspepsia. Journal of Gastroenterology and Hepatology, 25, S122-128. doi:10.1111/j.1440-1746.2009.06218.x

[18] Hongo, M., Miwa, H., Kusano, M., J-FAST group. (2011) Effect of rabeprazole treatment on health-related quality of life and symptoms in patients with reflux esophagitis: a prospective multicenter observational study in Japan. Journal of Gastroenterology, 46, 297-304. doi:10.1007/s00535-010-0342-1

[19] Thomson, A.B., Barkun, A.N., Armstrong, D., et al. (2003) The prevalence of clinically significant endoscopic findings in primary care patients with uninvestigated dyspepsia: the Canadian Adult Dyspepsia Empiric Treatment-Prompt Endoscopy (CADET-PE) study. Alimentary Pharmacology \& Therapeutics, 17, 14811491.doi:10.1046/j.1365-2036.2003.01646.x

[20] The Japanese Society of Gastroenterology. GERD management guideline. (2009) The Japanese Society of Gastroenterology, Tokyo,(in Japanese)

[21] Lin, C.C., Wang, Y.Y., Wang, K.L., et al. (2009) Association of heartburn and laryngopharyngeal symptoms with endoscopic reflux esophagitis, smoking, and drinking. Otolaryngology—Head and Neck Surgery, 141, 264-271. doi:10.1016/j.otohns.2009.05.017

[22] Belafsky, P.C., Postma, G.N. and Koufman, J.A. (2002) Validity and reliability of the reflux symptom index (RSI). Journal of Voice, 16, 274-277. doi:10.1016/S0892-1997(02)00097-8

[23] Hays, J.T., Croghan, I.T., Baker, C.L., Cappelleri, J.C. and Bushmakin, AG. (2010) Changes in health-related quality of life with smoking cessation treatment. Eur J Public Health. 30 September 2010. [Epub ahead of print]

[24] Jang, A.S., Park, S.W., Kim, D.J., et al. (2010) Effects of smoking cessation on airflow obstruction and quality of life in asthmatic smokers. Allergy, Asthma and Immunology Research, 2, 254-259. doi:10.4168/aair.2010.2.4.254 